\title{
Esquizofrenia e intervención familiar en España: Una revisión de las intervenciones psicológicas de los últimos diez años.
}

\author{
Noelia Navarro Gómez \\ Universidad de Almería
}

(Recibido el 14 febrero 2013; Aceptado el 28 mayo 2013)

RESUMEN: La necesidad de asumir una perspectiva familiar en el abordaje terapéutico de la esquizofrenia, ha desembocado en un aumento creciente de la literatura respecto al tópico. Se presenta una revisión de los artículos disponibles en castellano acerca de la intervención familiar en las 32 primeras revistas del Índice de Impacto de las Revistas Españolas de Ciencias Sociales para el año 2011(IN-RECS, Impact Index of Spanish Social Sciences Journals) durante el periodo 2001-2011. Los resultados se comparan con el total de artículos disponibles en a terapéutica de la esquizofrenia a nivel psicológico, sugiriendo la necesidad de seguir desarrollando este tipo de procedimientos que incorporen a la familia, de probada eficacia.

Palabras clave: terapia, familia, psicología, esquizofrenia.

Schizophrenia and family intervention in Spain: A review of psychological interventions for the last ten years.

\begin{abstract}
The need to take a family perspective in the therapeutic approach to schizophrenia, has led to an increasing amount of literature on the topic. A review of articles available in Spanish about family intervention in the first 32 magazines of the Impact Index of Spanish Journals of Social Sciences (IN- RECS) for 2011 during the period 2001-2011 is presented. The results are compared with the total number of articles in a therapeutic schizophrenia psychological level, suggesting the need to further develop these procedures that incorporate family of tested effectiveness.
\end{abstract}

Keywords: therapy, family, psychology, schizophrenia.

En las últimas décadas, aunque de forma incipiente parece haberse desarrollado un creciente interés hacia el papel que desempeñan las relaciones familiares en los trastornos del espectro esquizofrénico. Posiblemente, el proceso de desinstitucionalización de los hospitales psiquiátricos, la reducción en el tiempo de ingresos, o el considerar que el individuo es un producto de sus pautas de interacción hayan propiciado esta focalización de la atención en las familias, abordadas inicialmente de manera indirecta.

Asumir el abordaje a nivel familiar, es asumir la posibilidad de cambio y mejora, valorando la crisis familiar que este diagnóstico acarrea, el rol que desem- 
peña en su pronóstico, y en definitiva, asumiendo la interdependencia de la persona con su contexto, en el que inevitablemente, la familia quedaría enmarcada.

Se estima que la prevalencia de la esquizofrenia a lo largo de la vida está entre el 0,7 y el 1,5\%. En nuestro país, la incidencia estimada es de 0,8 casos por cada diez mil habitantes, lo que concuerda con los estudios de la Organización Mundial de la Salud, para los países europeos (Ayuso-Mateos, et al., 2006). Con respecto a la prevalencia estimada, la cifra es de 3 y de 2,86 por cada mil habitantes por año para hombres y mujeres respectivamente, con la consiguiente carga extra que este diagnóstico supone desde el punto de vista personal, familiar, social y económico.

Los costes que representan el tratamiento y cuidado de estos pacientes son particularmente elevados, representando un problema sanitario de primera magnitud, que incluiría los gastos derivados de los dispositivos asistenciales empleados (son pacientes de alta dependencia), el coste del tratamiento y el hecho de que se les otorgue una incapacidad que obstaculiza el acceso al mundo laboral. De hecho, los trastornos mentales suponen la segunda causa de baja laboral en España y el tercer grupo de enfermedades que genera más gasto al sistema sanitario público, suponiendo un coste económico de 7.019 millones de euros, de los cuales los costes médicos directos supusieron el $36.9 \%$, los costes asociados a cuidados informales el $17.7 \%$ y los costes por baja laboral el $42.7 \%$ (Moreno et al., 2009). Es también destacable, el hecho de que este tipo de trastornos constituyen el cuarto motivo de requerimiento de cuidados proporcionados por familiares y personal no profesional.

Por otro lado, en la literatura se manifiestan las limitaciones del sistema para atender de una forma adecuada a las necesidades e inquietudes de los familiares, lo que unido a la escasa información que tienen disponible, junto al estigma inherente a este tipo de trastornos, los coloca en una situación de indefensión con alto riesgo de exclusión social.

Esta notoria necesidad de implicar a los familiares en la intervención, clave en el abordaje terapéutico, ha propiciado esta revisión, con el objetivo de proporcionar una perspectiva general de las aportaciones surgidas en el panorama español en este tipo de intervenciones en la última década. Se trataría de ver el calado que ha tenido el desarrollo de la terapia familiar en nuestro país, y los posibles avances que hayan podido surgir al respecto, recopilando los esfuerzos que la investigación psicológica española ha realizado en la valoración de la familia y sus aportaciones para la mejora de este tipo de pacientes.

\section{MÉTODO}

\section{Materiales}

Se trabajó con las 32 primeras revistas correspondientes a los dos primeros cuartiles de la categoría de Psicología del Índice de Impacto de las Revistas Españolas de Ciencias Sociales (IN-RECS, Impact Index of Spanish Social Scienc- 
es Journals) para el año 2011, en el que se publico el último índice (ver tabla 1).

\section{Procedimiento}

Para la revisión, se recurrió a las propias web de las revistas, y se introdujeron en los correspondientes buscadores los términos "esquizofrenia" y "familia", además de los criterios temporales de búsqueda (publicaciones de los años comprendidos entre 2001 y 2011) analizándose los resultados que la búsqueda proporcionaba. Además, para producir resultados adicionales se introdujeron estos mismos criterios en la base de datos "Psicodoc. Se seleccionaron únicamente, aquellos resultados que abordaran las intervenciones a nivel psicológico y familiar del trastorno. Posteriormente, se revisaron en estas revistas todos los artículos disponibles publicados en esas fechas, relativos a cualquier procedimiento en la intervención psicológica.

\section{Tabla 1. Cuartiles 1 y 2 del Índice de Impacto de las Revistas Españolas de Ciencias Sociales (Psicología)}

\begin{tabular}{|c|c|c|c|}
\hline Cuartil & Posicion & Titulo dE LA REVISTA & $\begin{array}{l}\text { INDICE IMPACTO } \\
2011\end{array}$ \\
\hline \multirow[t]{16}{*}{$1^{\circ}$} & 1 & International journal of clinical and health psychology & 2.327 \\
\hline & 2 & Revista de psicodidáctica & 2.256 \\
\hline & 3 & $\begin{array}{l}\text { Revista de psicopatología y psicología clínica: revista oficial de la Asociación Española de Psicología } \\
\text { Clínica y Psicopatología AEPCP }\end{array}$ & 1.311 \\
\hline & 4 & Psicothema & 1.244 \\
\hline & 5 & Revista de psicología del deporte & 1.216 \\
\hline & 6 & Spanish journal of psychology & 1.005 \\
\hline & 7 & International journal of psychology and psychological therapy & 0.979 \\
\hline & 8 & Adicciones: Revista de socidrogalcohol & 0.711 \\
\hline & 9 & Papeles del psicólogo: revista del Colegio Oficial de Psicólogos & 0.671 \\
\hline & 10 & Ansiedad y estrés & 0.667 \\
\hline & 11 & Infancia y Aprendizaje: Journal for the Study of Education and Development & 0.651 \\
\hline & 12 & Cuadernos de psicología del deporte & 0.633 \\
\hline & 13 & Anales de psicología & 0.602 \\
\hline & 14 & $\begin{array}{l}\text { Psicología conductual = behavioral psychology: Revista internacional de psicología clínica y de la } \\
\text { salud }\end{array}$ & 0.589 \\
\hline & 15 & Actas españolas de psiquiatría & 0.452 \\
\hline & 16 & Cadernos de psicoloxía & 0.444 \\
\hline \multirow[t]{8}{*}{$2^{\circ}$} & 17 & Revista de Psicología Social & 0.420 \\
\hline & 18 & Psicológica: Revista de metodología y psicología experimental & 0.418 \\
\hline & 19 & Estudios de Psicología & 0.417 \\
\hline & 20 & Boletín de psicología & 0.380 \\
\hline & 21 & Acción Psicológica & 0.362 \\
\hline & 22 & Revista de psicología del trabajo y de las organizaciones & 0.288 \\
\hline & 23 & Electronic journal of research in educational psychology & 0.287 \\
\hline & 24 & Clínica y salud: Revista de psicología clínica y salud & 0.275 \\
\hline
\end{tabular}




\begin{tabular}{|l|l|l|}
\hline 25 & Psicología educativa & 0.270 \\
\hline \hline 26 & Revista Española de Orientación y Psicopedagogía & 0.248 \\
\hline 27 & Anuario de psicología & 0.213 \\
\hline 28 & Escritos de psicología & 0.205 \\
\hline 29 & REMA (Revista Electrónica de Metodología Aplicada) & 0.200 \\
\hline 30 & Salud y drogas & 0.182 \\
\hline 30 & Trastornos adictivos: Organo Oficial de la Sociedad española de Toxicomanías & 0.182 \\
\hline 32 & Papeles de Trabajo sobre Cultura, Educación y Desarrollo Humano & 0.167 \\
\hline
\end{tabular}

\section{RESULTADOS}

Se presenta una tabla resumen de las revistas consultadas, el número de resultados encontrados tanto para la terapia psicológica en todas sus modalidades de intervención (excluyendo la terapia familiar), como las intervenciones a nivel familiar, añadiéndose en estos casos, los autores y fecha de publicación junto con un breve resumen del mismo. Del mismo modo, se expone la distribución de los artículos sobre terapia familiar por años. 


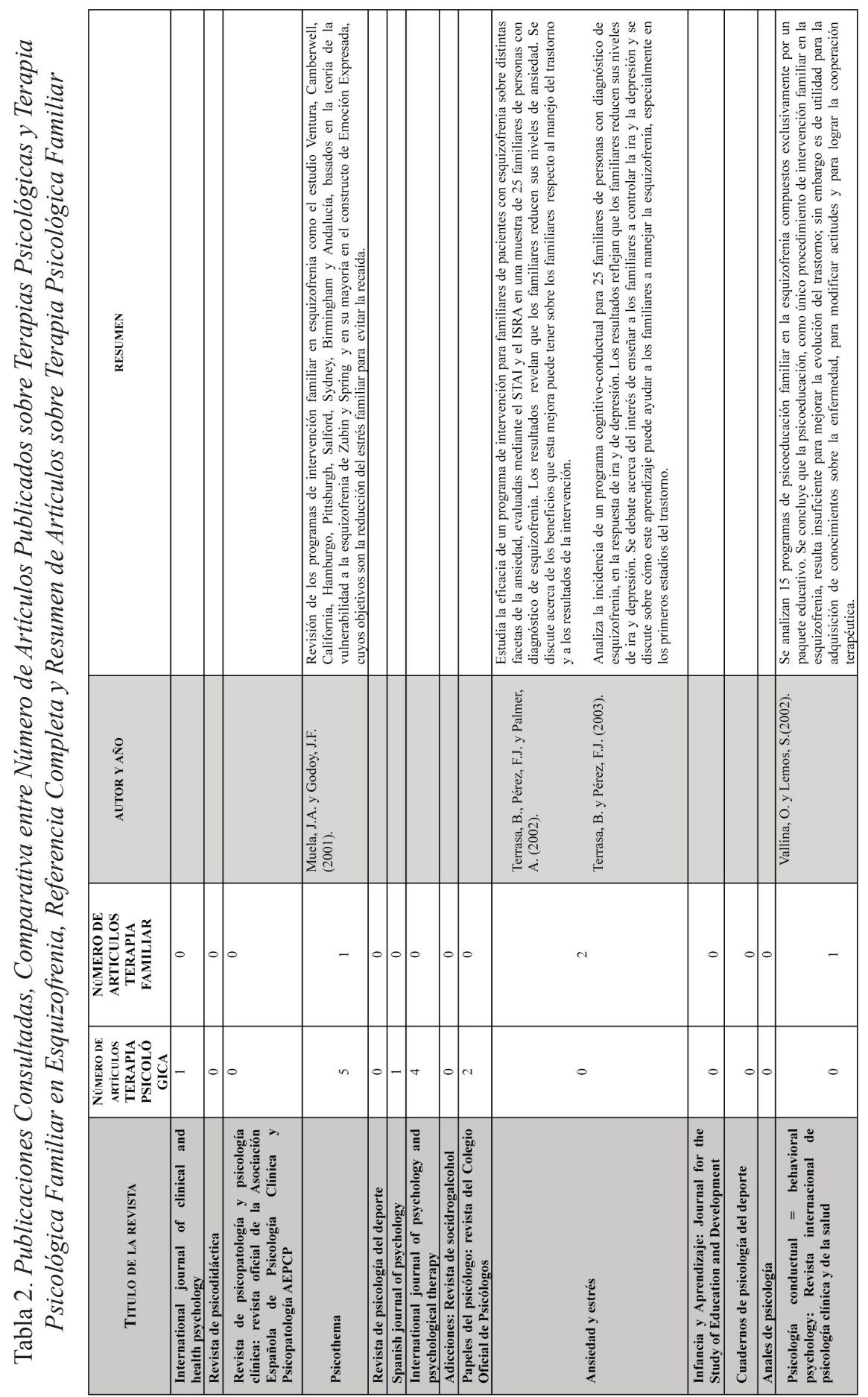

(CPsy, Soc, \& Educ, 2013, Vol.5, No2 


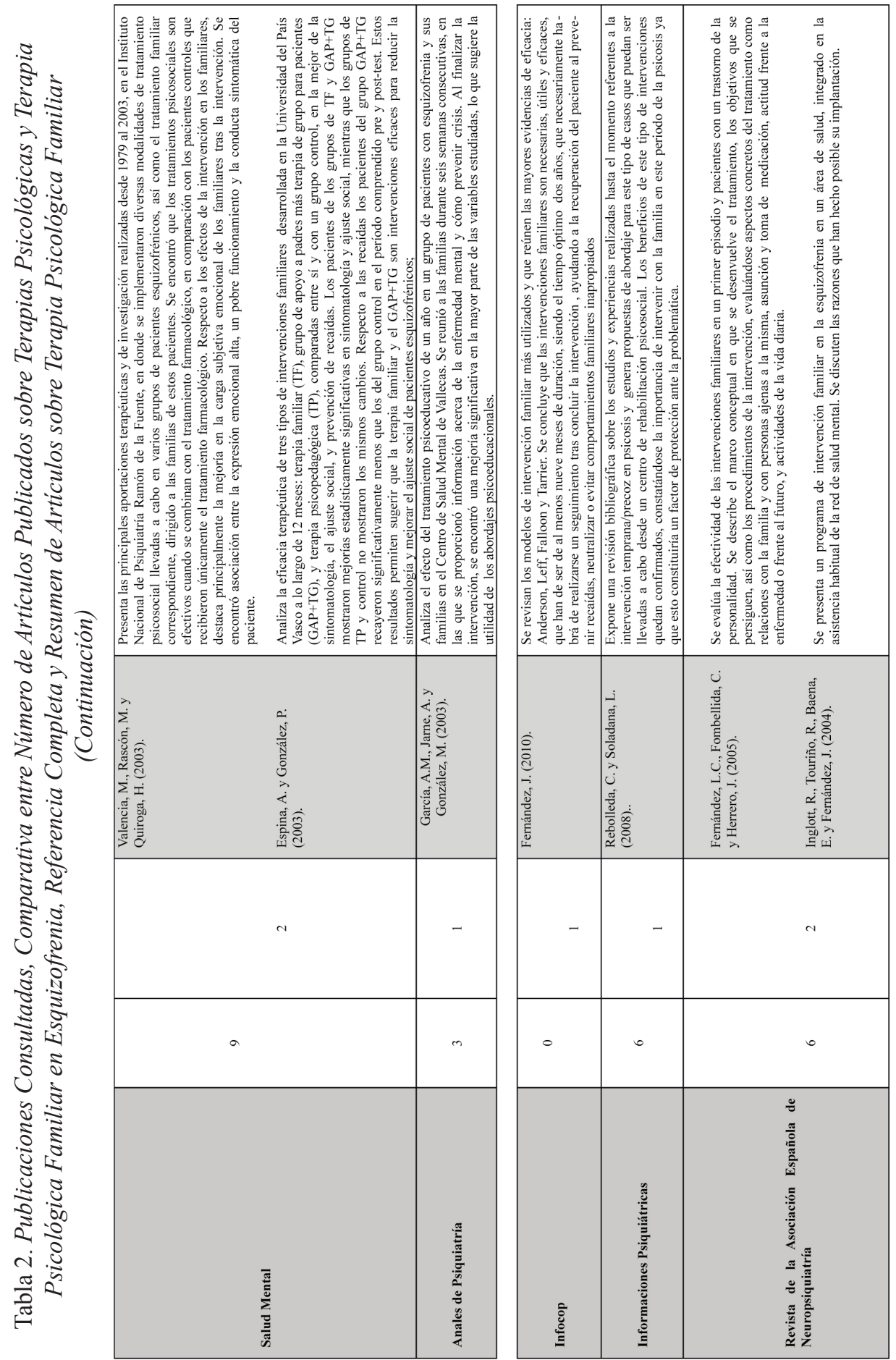

(c) Psy, Soc, \& Educ, 2013, Vol.5, N² 
NAVARRO. Inervención familiar en esquizofrenia

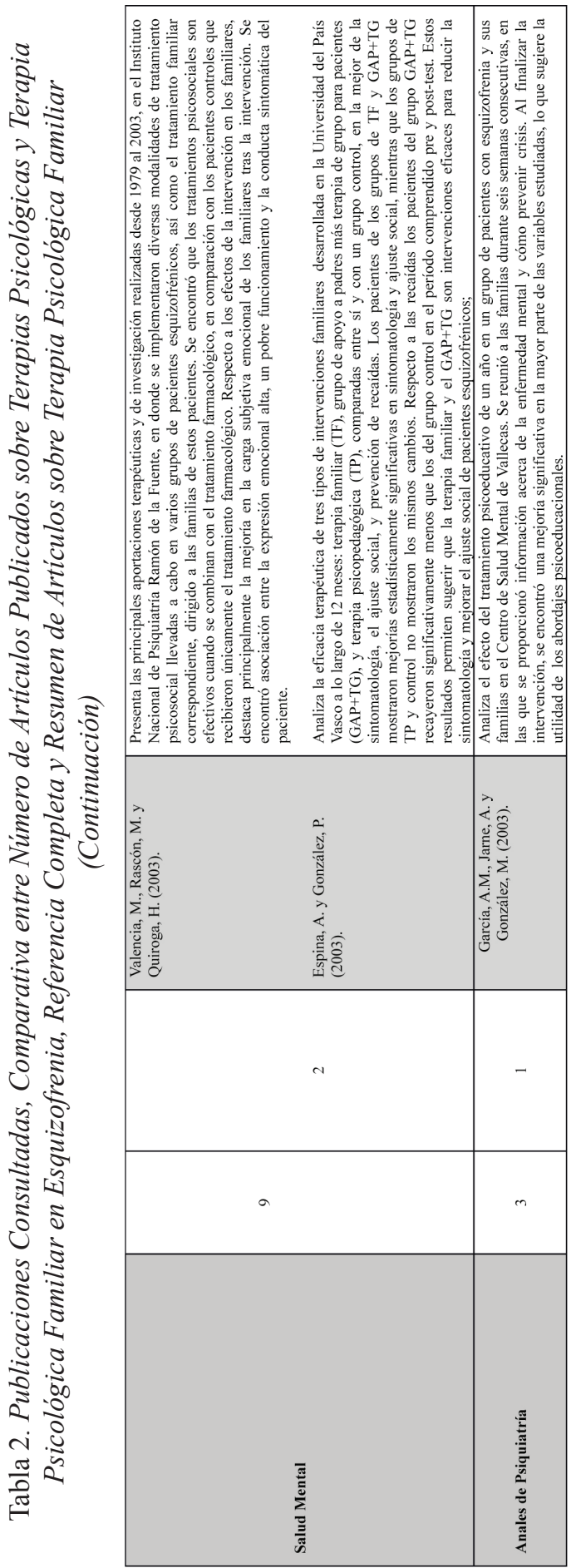

\begin{tabular}{|c|c|c|c|}
\hline 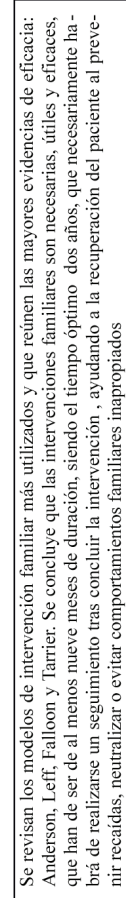 & 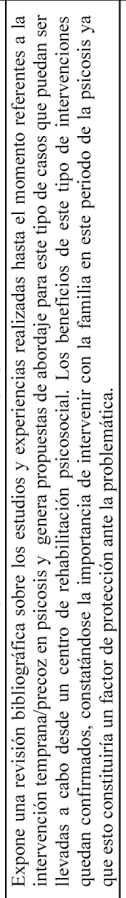 & 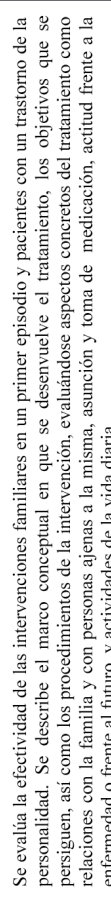 & 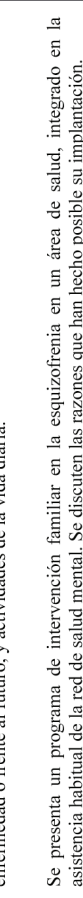 \\
\hline 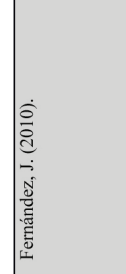 & 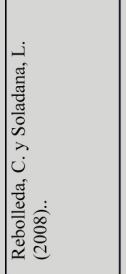 & 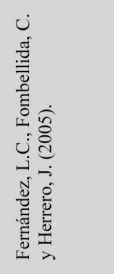 & 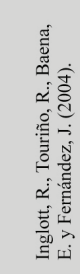 \\
\hline- & - & & $a$ \\
\hline 0 & 0 & & 0 \\
\hline 总 & 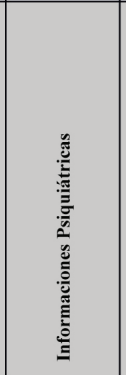 & & 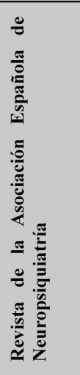 \\
\hline
\end{tabular}

(C) Psy, Soc, \& Educ, 2013, Vol.5, No2 
Tabla 3. Distribución por Años de las Publicaciones sobre Terapias Psicológicas Familiares para la Esquizofrenia

\begin{tabular}{|l|c|}
\hline Año & $N^{o}$ de artículos \\
\hline 2001 & 2 \\
\hline 2002 & 2 \\
\hline 2003 & 4 \\
\hline 2004 & 1 \\
\hline 2005 & 3 \\
\hline 2006 & 0 \\
\hline 2007 & 0 \\
\hline 2008 & 3 \\
\hline 2009 & 0 \\
\hline 2010 & 1 \\
\hline 2011 & 1 \\
\hline
\end{tabular}

La temática de los artículos revisados se ilustra en la Figura 1.

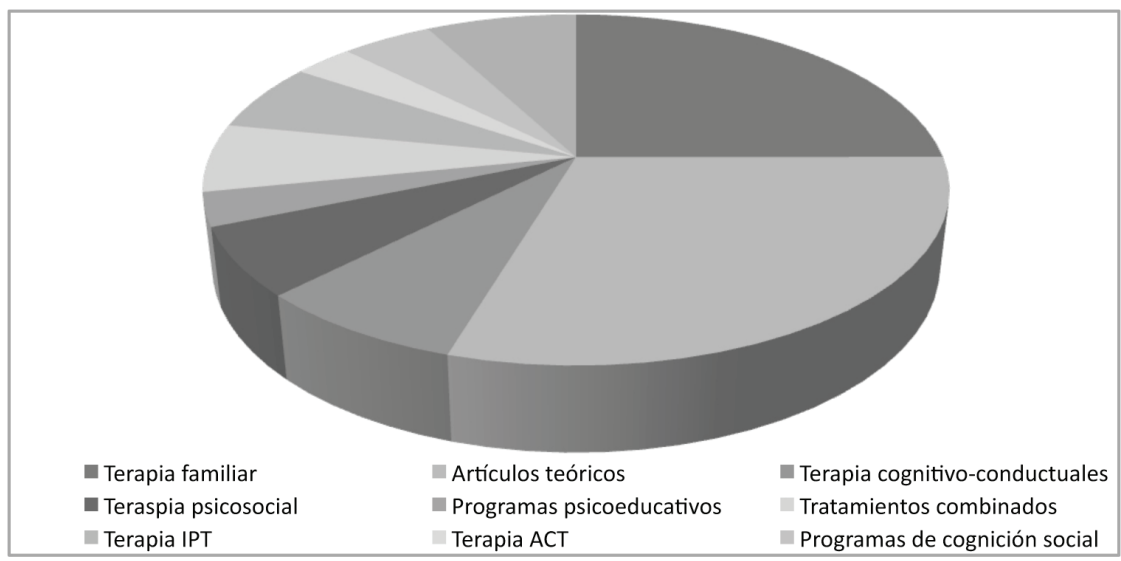

Figura 1. Distribución de los artículos en función de su contenido.

\section{DISCUSIÓN}

La revisión de los artículos relacionados con los tópicos "esquizofrenia" y "familias", permite concluir que si bien, la literatura psicológica respecto al tema es prolífica y abundante en líneas generales dado el gran interés que ha generado el estudio del papel de la familia en este tipo de trastornos, no parece ser así 
en la literatura española de los últimos diez años, donde las revistas de impacto no han dado gran importancia en materia de investigación a este proceder en el abordaje psicológico. Así, de las revistas revisadas correspondientes a los dos primeros cuartiles del Índice de Impacto de las Revistas Españolas de Ciencias Sociales (Psicología) sólo 4 aludirían a esta temática, siendo 6 el número total de artículos, frente a los 16 artículos encontrados en alusión a otros tipos de proceder en la intervención psicológica en este trastorno, bien de corte aplicado, bien a nivel teórico. En relación a las revistas y la intervención a nivel familiar, Ansiedad y estrés y Actas españolas de psiquiatría, cuentan cada una de ellas con 2 artículos, siendo el resto de revistas, Psicothema y Psicología Conductual. Los artículos encontrados a través de la bases de datos Psicodoc, (un total de 10 y 28 para la intervención a nivel familiar, y para otros tipos de intervención psicológica, respectivamente) proceden de las revistas Apuntes de Psicología, Salud Mental, Anales de Psiquiatría, Infocop, Informaciones Psiquiátricas y Revista de la Asociación Española de Neuropsiquiatría.

Profundizando más en el contenido de los artículos encontrados en los que se contemple la actuación a nivel familiar, en su mayoría presentan los resultados de la implantación de algún tipo de intervención, generalmente, de carácter psicoeducativo, centrándose uno de ellos en las experiencias de los profesionales sanitarios; otro estudio, compara la eficacia de tres tipos de intervenciones a nivel familiar y dos de ellos se centran en la intervención familiar temprana, en los primeros episodios psicóticos. Además, los dos artículos de la revista "Ansiedad y estrés" analizan exclusivamente el papel de la intervención familiar en la mejora del bienestar de los familiares, incidiendo en la disminución de los niveles de ira, depresión y ansiedad, no valorando por el contrario, los posibles efectos que el programa ejerce sobre los pacientes. Otra parte de los artículos encontrados (un total de 4) realizan revisiones centrándose en los siguientes aspectos: las actuaciones históricamente más utilizadas en la intervención familiar, centrándose en la teoría de vulnerabilidad a la esquizofrenia y emoción expresada, los programas que mayores niveles de eficacia han reportado (Anderson, Leff, Falloon y Tarrier), la revisión bibliográfica sobre los estudios y experiencias realizadas en la intervención temprana en psicosis y la revisión de los datos empíricos sobre los programas psicoeducativos familiares. En líneas generales, podemos concluir que las intervenciones familiares muestran resultados positivos, siendo eficaces en la reducción de las exacerbaciones en la esquizofrenia, e incluso mejorando el funcionamiento social y reduciendo la carga familiar.

Pese a constituir la cuarta parte de los resultados encontrados, el escaso número de artículos publicados en torno al tópico (una media de 1,6 por año) hace plantearse la necesidad de seguir trabajando en este tipo de cuestiones. Posiblemente, se haya producido un estancamiento en este tipo de intervenciones, y aunque los efectos encontrados sean beneficiosos, los programas que trabajan a nivel psicoeducativo han mostrado sus límites. Dada la escasez de artículos, y los procedimientos que se utilizan, podría entreverse que la investigación al respecto no ha mostrado grandes avances o al menos, modificaciones, en los 
últimos diez años en el panorama español, no surgiendo en la prensa de revistas nuevas propuestas para la intervención familiar más allá de las que se han venido usando tradicionalmente. En este punto sería conveniente señalar que si bien, se ha profundizado en el conocimiento acerca de la relación entre familia y esquizofrenia, no es este el caso de las teorías que han inspirado esas investigaciones, basando la mayor parte de estas en teorías formuladas en los años 50, sin haberse producido grandes avances conceptuales (Merino y Pereira, 1990). Aunque se sigan utilizando este tipo de intervenciones, no son objeto de la investigación, sino algo más exclusivo de los procederes dentro de la práctica terapéutica. No obstante, conviene señalar, que en líneas generales, la investigación en el tratamiento a nivel psicológico en la esquizofrenia no ha sido demasiado productiva en cuanto a número de artículos se refiere en estos últimos años, como se entrevé en los resultados de la búsqueda. Entre las explicaciones plausibles, destacar la resistencia de creencias entre los profesionales en lo relativo al tratamiento farmacológico como única o principal vía de abordaje terapéutico y la asunción del modelo biomédico, no desarrollándose hasta hace pocos años, estrategias y terapias psicológicas de probada eficacia. Estos artículos, serían en su mayoría de corte teórico (19), tratando el resto estudios sobre la implantación de programas de corte cognitivo (5), intervención psicosocial (4), psicoeducativa (2), tratamientos combinados (4), terapia psicológica integrada de Roder (4), terapia de Aceptación y Compromiso (2), aplicación de programas de cognición social (3), musicoterapia (1), terapia con animales (1) o tratamiento de otros aspectos comórbidos, como la adicción al tabaquismo, la obesidad o la ansiedad.

Sin embargo, ha de señalarse que este revisión sólo se ha basado en la investigación española desarrollada en los últimos diez años, y únicamente recurriendo a fuentes como revistas de investigación, no habiéndose revisado los libros publicados, o las investigaciones que hayan podido surgir en otros países, con lo que los resultados obtenidos podrían estar subestimando la realidad, y deben tenerse en cuenta como datos exploratorios.

Pese a estas limitaciones, cabría preguntarse las causas que dificultan una mayor difusión de este tipo de programas, como podrían ser problemas de adherencia de los familiares, o resistencia a la terapia, edad avanzada o problemas de salud física, falta de recursos o incluso la fase en la que se inicie la intervención, (la intervención temprana propiciaría una mayor adherencia). Del mismo modo, pese a disponer de evidencias empíricas suficientes para su implantación, es llamativa la resistencia a su elección en ciertos dispositivos sanitarios, lo que podría ser explicado atendiendo a razones como la escasa confianza por parte de algunos profesionales de los servicios de salud mental en las evidencias científicas, del peso mayor de otras teorías tradicionales de escasa compatiblidad con este tipo de procederes, de la no valoración del papel de la familia como aliada terapéutica y recurso asistencial o del alto nivel requerido para la formación y cualificación en los equipos, ligado al cambio exigido en los procedimientos de intervención (Hahlweg y Wiedemann, 1999; Lehman, 2000).

En cualquier caso, deberá tenerse en cuenta carácter idiosincrásico que debe 
subyacer a cualquier intervención, así como la necesidad de contemplar las preferencias de cada paciente y familias, grandes aliados que trabajan para movilizar todos los apoyos disponibles y por fortalecer mecanismos de afrontamiento naturales. Igualmente, el concepto de familia debería ampliarse para incluir tanto las relaciones maritales como fraternales y paternales, así como identificar mejores vías para acompasar usuario y tratamiento, y superar los posibles obstáculos de implantación.

La familia supone un foco potencial en la intervención, como elemento natural y de primer orden dentro del contexto en el que se desenvuelve la vida del paciente; sólo trabajando a todos los niveles, y por ende, implicando a la familias teniendo en cuenta las particularidades de cada caso, estaremos en condiciones de garantizar una intervención integral, necesaria para el propio avance de la disciplina en el desarrollo de herramientas, técnicas y estrategias de intervención eficaces.

\section{REFERENCIAS}

Ayuso-Mateos, J.L., Gutiérrez-Recacha, P., Haro, J.M y Chisholm, D. (2006). Estimating the prevalence of schizophrenia in Spain using a disease model. Schizophrenia Research, 86, 194-201.

De la Higuera, J. (2005). Psicoeducación familiar en la esquizofrenia: de los datos empíricos a la realidad clínica. Apuntes de Psicología, 23, 275-292.

De la Higuera, J. y Ramírez, R.M. (2011).Implementación de programas de psicoeducación familiar en la práctica clínica: análisis de una experiencia. Apuntes de Psicología, 29,71-85.

Espina, A. y González, P. (2003). Intervenciones familiares en la esquizofrenia. Cambios en la sintomatología y el ajuste social. Salud Mental, 26, 51-58.

Fernández, J. (2010). Modelos de intervención con familias de personas afectadas por una enfermedad mental grave y duradera. Infocop, 47,6-10.

Fernández, L.C., Fombellida, C. y Herrero, J. (2005).Evaluación de un programa de intervención familiar en pacientes psicóticos y con trastornos graves de la personalidad. Revista de la Asociación Española de Neuropsiquiatría, 94,7-18.

García, A.M., Jarne, A. y González, M. (2003). Tratamiento psicoeducativo en pacientes con esquizofrenia. Anales de psiquiatría, 19, 137-142.Moreno, E. y Moreno, S. (2007). El papel del Terapeuta Ocupacional en un grupo de Psicoeducación de familias de personas con esquizofrenia. Terapia ocupacional: Revista informativa de la Asociación Profesional Española de Terapeutas Ocupacionales, 42, 48-52.

Godoy, J.F. y Muela, J.A. (2001). Programa de intervención familiar en esquizofrenia: dos años de seguimiento del estudio de Andalucía. Apuntes de Psicología, 19, 421-430.

González, J. y Rodríguez, A. (2010). Programas de rehabilitación psicosocial en la atención comunitaria a las personas con psicosis. Clínica y Salud, 21, 
319-332.

Hahlweg, K. y Wiedeman, G. (1999). Principles and results of family therapy in schizophrenia. European Archives of Psychiatry and Clinical Neurosciences, 249, 108-115.

Hurtado, G., Roger, M., Alcoverro, O. y López, N. (2008).Una experiencia con grupos multifamiliares en pacientes con esquizofrenia. Actas españolas de Psiquiatría, 36, 120-122.

Inglott, R., Touriño, R., Baena, E. y Fernández, J. (2004). Intervención familiar en la esquizofrenia: su diseminación en un área de salud. Revista de la Asociación Española de Neuropsiquiatría, 92,9-23.

Leal, M.I., Sales, R., Ibáñez, E. y Leal, C. (2008). Valoración de la sobrecarga en cuidadores informales de pacientes con esquizofrenia antes y después de un programa psicoeducativo. Actas Españolas de Psiquiatría, 36, 63-69.

Lehman, A.F. (2000). Commentary: What happens to psychosocial treatments on the way to clinic? Schizophrenia Bulletin, 26, 137-139.

Moreno, J.O., López Bastida, J., Montejo-González, A.L., Osuna-Guerrero, R., y Duque-González, B. (2009). The socioeconomic costs of mental illness in Spain. European Journal of Health Economics, 10, 361-369.

Merino, H. y Pereira, M. (1990). Familia y esquizofrenia: una revisión desde el punto de vista de interacción familiar. Anales de Psicología, 6, 11-20.

Muela, J.A. y Godoy, J.F. (2001). Programas actuales de intervención familiar en esquizofrenia. Psicothema, 13,1-6.

Rebolleda, C. y Soladana, L. (2008). Propuestas de abordaje en la intervención precoz de la psicosis con usuarios de un CRPS y sus familias. Informaciones Psiquiátricas 194,343-369.

Terrasa, B. y Pérez, F.J. (2003). Ira y depresión en familias de personas con esquizofrenia: incidencia de un programa de intervención familiar. Ansiedad y estrés, 9,35-48.

Terrasa, B., Pérez, F.J. y Palmer, A. (2002). Ansiedad en familiares de esquizofrénicos: eficacia de un programa de intervención familiar. Ansiedad y estrés, 8, 143-155.

Valencia, M., Rascón, M. y Quiroga, H. (2003). Aportaciones de la investigación respecto al tratamiento psicosocial y familiar de pacientes con esquizofrenia. Salud Mental. 26, 1-18.

Vallina, O. y Lemos, S. (2002). Los programas de educación familiar en la esquizofrenia. Psicología conductual = behavioral psychology: Revista internacional de psicología clínica y de la salud, 2, 287-304. 